Instituto Internacional de Investigación y Desarrollo Tecnológico Educativo INDTEC, C.A.

DOI: https://doi.org/10.29394/Scientific.issn.2542-2987.2020.5.16.20.372-383

OAI-PMH: http://www.indteca.com/ojs/index.php/Revista Scientific/oai

Ensayo Original / Original Essay

\title{
Efecto Zeigarnik y funciones ejecutivas en educación superior para las ciencias clínicas
}

Autora: Ximena Paz Martínez Oportus

Universidad Mayor, UM

ximena.martinez@umayor.cl

Temuco, Chile

https://orcid.org/0000-0002-7130-1316

\section{Resumen}

En la educación superior de hoy, los docentes, intentan formar a sus estudiantes en tareas y competencias, con enfoque en el "saber hacer", de manera de agilizar los procesos de enseñanza aprendizaje. De esta forma, cabe la inquietud respecto a la posibilidad de potenciar la creación de saberes, toma de decisiones y habilidades a través de la parcialización de las tareas, basado en el Efecto Zeigarnik, declarado por Zeigarnik (1927); avalado por Ovsiankina (1928); y su impacto en las funciones ejecutivas, con el objetivo final de dar soporte a nuevas herramientas para la educación en ciencias clínicas propuestas por Diamond (2013). Desde este contexto de análisis, se vierten algunas reflexiones con pretensiones de instalar posibles dilemas a considerar para la validación de la necesidad de repensar la ejecución del proceso de enseñanza aprendizaje en el aula de educación superior.

Palabras clave: educación superior; aprendizaje; docente.

Cómo citar este ensayo:

Martínez, X. (2020). Efecto Zeigarnik y funciones ejecutivas en educación superior para las ciencias clínicas. Revista Scientific, 5(16), 372-383, e-ISSN: 2542-2987. Recuperado de: https://doi.org/10.29394/Scientific.issn.2542-2987.2020.5.16.20.372-383

Fecha de Recepción:

04-12-2019
Fecha de Aceptación:

31-03-2020
Fecha de Publicación:

05-05-2020 


\title{
Zeigarnik effect and executive functions in higher education for clinical sciences
}

\begin{abstract}
In today's higher education, teachers try to train their students in tasks and competences, with a focus on "knowing how", in order to streamline the teaching-learning processes. Thus, there is concern about the possibility of enhancing the creation of knowledge, decision-making and skills through the partialization of tasks, based on the Zeigarnik Effect, declared by Zeigarnik (1927); endorsed by Ovsiankina (1928); and its impact on executive functions, with the ultimate goal of supporting new tools for clinical science education proposed by Diamond (2013). From this context of analysis, some reflections are presented with the aim of installing possible dilemmas to consider for the validation of the need to rethink the execution of the teaching-learning process in the higher education classroom.
\end{abstract}

Keywords: higher education; learning; teacher.

How to cite this essay:

Martínez, X. (2020). Zeigarnik effect and executive functions in higher education for clinical sciences. Revista Scientific, 5(16), 372-383, e-ISSN: 2542-2987. Recovered from: https://doi.org/10.29394/Scientific.issn.2542-2987.2020.5.16.20.372-383
Date Received:

04-12-2019
Date Acceptance:

31-03-2020
Date Publication:

05-05-2020 


\section{Introducción}

En la educación superior para las ciencias clínicas, nos encontramos ante la necesidad de gestionar de modo idóneo la tarea de atención en el aula, sobre todo en actividades práctico-clínicas que involucran la toma de decisiones. Es de esta manera, en que se generan las habilidades finales de intervenciones clínicas, quirúrgicas y procedimentales, a las que se verá expuesto el estudiante en la realidad laboral. Bajo esta mirada de un desempeño aprehendido de manera sólida y transferible, nace la pregunta sobre la necesidad de robustecer el proceso de enseñanza aprendizaje, no solamente contextualizado, sino también, en base a los avances de la neurociencia de la educación, en un aprendizaje escalonado y significativo.

Es así, como aparece en el análisis, la fenomenología de la atención y se vincula ésta con la intención, el pensamiento, la voluntad, el interés y la creatividad. Aparece en este análisis el Efecto Zeigarnik (tendencia a recordar tareas inacabadas o interrumpidas con mayor facilidad que las que han sido completadas), sobre el que se despliegan algunas reflexiones en virtud de poder ser transferido al aula. Esto en virtud de brindar a la academia soporte a nuevas herramientas para la educación en ciencias clínicas

\section{Desarrollo}

A modo de contextualización, cabe reflexionar sobre el stress fisiológico nombrado por Celis, Bustamante, Cabrera, Cabrera, Alarcón y Monge (2001): que se suscita en el aula y la tensión que induce cada actividad de aprendizajes, produce dificultad en el proceso de enseñanza aprendizaje, donde cada cambio en las situaciones a la que el estudiante se enfrenta, determina cierto nivel de sobrecarga para cumplir con las demandas de cada currículum. Estás demandas, según Cabanach, Souto-Gestal y Franco (2016): generan estrés en el proceso no solo en la interacción entre las personas, como con en el entorno. En experimentos clásicos, Zeigarnik (1927); y 
Ovsiankina (1928): utilizaron recuperación y reanudación de tareas, encontraron que una tarea interrumpida que se reanuda espontáneamente, es más probable que sea recordada que una tarea que ha sido completada.

Estas tendencias, son mayores cuando la interrupción se produce cerca de la finalización de la meta. Por lo tanto, hace reflexionar sobre la importancia de reconsiderar cuales serían los factores que potencian esta respuesta, ya que, en las actividades prácticas en el aula de educación en las ciencias clínica, pareciera ser que se repitiera esta estructura fenomenológica en relación al desempeño ante las tareas.

Pensando en las intervenciones en aula, la interrupción de tareas es la metodología que pudiese inducir a nuestros estudiantes a una mejor toma de decisiones en el ámbito clínico y procedimental; basado en esto, también impactar en la variante motivacional como motor del proceso de enseñanza aprendizaje. Sabemos que en el desarrollo de la sociedad el aprendizaje, el estudio y la educación juegan un papel fundamental. Pero también sabemos que las características propias de la sociedad van cambiando con el tiempo debido a la evolución de sus componentes, por lo que, si queremos que ese proceso de aprendizaje siga siendo efectivo, las metodologías deben adaptarse a las características de los nuevos individuos. Existen varios estudios en el área de desarrollo de negocios, como los de Li, Li y Chen (2018): que postulan que la autonomía laboral en los estudiantes se correlaciona con mayor motivación y creatividad en su desempeño, pero el efecto es más potente, si la supervisión es alta. Lo cual puede ser llevado al aula clínica, en relación por ejemplo a la parcialización y supervisión intermitente en actividades de simulación clínica, por ejemplo.

Los estudios han discutido durante mucho tiempo que la motivación intrínseca lleva a los individuos a ser curiosos, a tomar riesgos, a que rompa las rutinas y persista a pesar de los obstáculos, tal como indica Shin y Zhou (2017): lo cual pasa a ser un eje importante en el desempeño de los 
estudiantes, en la búsqueda de soluciones, en la toma de decisiones y en su estado de bienestar, durante el transcurso de su paso por la educación superior.

Si volvemos nuevamente a los estudios realizados en negocios y en los consumidores, en este sentido, Kupor y Tormala (2015): identifican una característica fundamental inherente a la interrupción temporal de una tarea, una exposición repetida a los estímulos de decisión, y encontrar que esta re exposición reduce la novedad subjetiva de los estímulos, lo cual modula la decisión de riesgo, esto podría verse reflejado en la toma de decisiones clínicas, y pudiese ser una impronta de bastante relevancia considerando dos puntos: primero, la supervisión en el desempeño de los estudiantes y segundo, al corregir la tarea, dada por una decisión tomada "en pasos", se tenderá a generar un aprendizaje significativo, lo cual inducirá una respuesta semiautomatizada en el estudiante en una situación real, de manera protocolarizada.

Además, estos autores, indican que hay un aumento de la curiosidad por la tarea, de acuerdo con Portilla y Leyva (2018): estos modelos se han utilizado en educación, como el uso metodologías basadas en la evaluación diagnóstica de las experiencias previas de aprendizaje de los estudiantes y las habilidades de pensamiento, parcializando la intervención pedagógica, concluyendo que todos los participantes describen una importante experiencia de reflexiones metacognitivas, necesarias para la generación del conocimiento.

Si nos enfocamos en el impacto que pudiese tener el factor emocional y motivacional, otros estudios como el de Halkjelsvik y Rise (2014): señalan que los participantes que realizaron una tarea no motivante optaron por el término de la tarea después de que la interrumpieron, incluso, en ausencia de una recompensa económica, con un deseo de completar per se, conclusión interesante si pensamos que nuestros estudiantes están expuestos a 
evaluaciones formativas y que la recompensa es la evaluación cuantitativa. Paralelamente, Wilkie (2016): sugiere que los estudiantes que perciben un desafío mayor en las tareas de las asignaturas de matemáticas, mejoraban su motivación, compromiso e interés, siendo un beneficio para el aprendizaje

Si abarcamos el área de turismo se describe la segmentación en tres escenas: 1). antes del viaje, 2). durante el viaje, y 3). después del viaje, para Hiramatsu, Sato, Ito, Hatano, Sato, Watanabe y Sasaki (2017): esto genera una parcialización sistemática en la interpretación de la experiencia. Esto nos permite repensar las técnicas de intervención en aula, respecto a que la interrupción de tareas que generen un desafío en el estudiante puede aumentar su motivación y además a estructurar el recuerdo de manera parcializada.

Si analizamos las funciones ejecutivas, descritas por Diamond (2013): vemos que son las encargadas de modular, regular y controlar los comportamientos, entre ellos la planificación, control inhibitorio, respuestas de adaptación, emociones y cogniciones encargadas del logro de metas cobra sentido, el hecho de tener en aula a los estudiantes con desafíos contantes y fuera de la monotonía. Estos mecanismos que integración que trabajan a diferentes tiempos y de diferente forma, permiten atraer y dinamizar los conocimientos, cogniciones, emociones y memoria, desde el pasado hacia el futuro, de manera de dar una mejor respuesta y solución a los desafíos, de manera novedosa, que de acuerdo con Verdejo-García y Bechara (2010): es lo que esperamos generar en docencia.

Con una nueva propuesta de intervención en aula, con actividades parcializadas, potenciaríamos la planificación y la resolución de problemas. Ambas funciones ejecutivas complejas, son nuestro foco en los estudiantes de educación superior que se desempeñan ya en actividades clínicas que requieren de dimensiones elevadas de metacognición. Es por esto, que cuando describimos la importancia de la motivación en el aula y en la actividad 
práctico clínica, no podemos independizarla de las emociones, como pivote central de la interpretación de la realidad.

Basado en esto, que surge la importancia de considerar que todos los aspectos relacionados con la emocionalidad, permiten que se genere una interconectividad entre varias regiones cerebrales, que participan activamente en el aprendizaje. Estas regiones implicadas en el aprendizaje y que se interconectan de manera inevitable, acorde con esto, Lang y Davis (2006); y Morgane, Galler y Mokler (2005): son la corteza prefrontal, el hipocampo, el hipotálamo y la amígdala Es por esta razón, que el estudiante o el individuo que aprende, al adquirir un nuevo conocimiento, estimula la totalidad de su cerebro en el proceso. Esto nos permite definir, estrategias de intervención motivadoras, desafiantes y novedosas.

Las nuevas propuestas de enseñanza centrada en el estudiante hacen hincapié en que cada individuo cognoscente experimenta una realidad única e irrepetible, y que tal como lo detallan Elizondo, Rodríguez y Rodríguez (2018): la cual, estará determinada por el contexto en el que se produzca el aprendizaje en combinación con la experiencia que desarrolla en el proceso. Es relevante, entonces, poner en discusión la importancia de parcializar el trabajo en aula, basado en la potencialización de la experiencia de aprendizaje, que considera de manera central, la obtención de los resultados de aprendizaje, la motivación del estudiante y el robustecimiento de los conocimientos, dando la posibilidad de fortalecer los procesos ejecutivos y potenciar la adecuada toma de decisiones.

Adherimos al concepto de que el cerebro humano, funciona según aquello que es cierto para él, además, sabemos que sin emoción no hay aprendizaje real. De hecho, toda situación problemática o de estrés conduce a un patrón de aprendizaje irreversible e involuntario, ya que nuestro cerebro registra y almacena sólo aquello que implique una utilidad, una amenaza o una muy grata evocación. 
Es decir, solamente recordamos lo que nos emociona. Tal como sugieren Manes y Niro (2014): emociones y aprendizaje, de este modo, prosiguen de la mano. Es por esta razón, que la retroalimentación, la supervisión y la interrupción de tareas, puede lograr que el individuo cree realidades con la percepción correcta e intencionada, de manera de generar estrategias significativas, a fin de que el modelo enseñanza-aprendizaje responda al desarrollo neurofisiológico del individuo. Para Pesántez (2020): las estrategias de innovación en aula, según describen algunos autores, no necesariamente pueden ser gestadas por aquellos con grandes dotes disciplinares, sino que se basan en la creatividad e interdisciplinariedad en que trabajan los cuerpos académicos, que logren un estado afectivo-cognitivo a través de un estado mental positivo, que como indica Schaufeli, Martínez, Marques, Salanova y Bakker (2002): facilita el interés y compromiso del estudiantado.

\section{Conclusiones}

En este ensayo proponemos que, las tareas interrumpidas, vigiladas y retroalimentadas, generan motivación, curiosidad y aprendizaje significativo en el estudiante, es por esta razón que se propone en algunas actividades de simulación clínica o de trabajo teórico práctico, donde se requiera planificación y resolución de problemas, bajo un desafió controlado, generar y potenciar el Efecto Zeigarnik, como proceso neurocognitivo de apoyo al aprendizaje, en el contexto, que además en instancias clínicas se puede generar un ambiente controlado de estados emocionales del estudiantado, de manera de encuadrarlos dentro de un contexto equilibrado de emocionalidad.

Comprendemos que, bajo ciertas condiciones, no completar una tarea puede en realidad aumentar la motivación de las personas para participar en la tarea, que la persona que realiza la tarea debe estar razonablemente cerca de completarla, tanto que pueda percibir que está a su alcance, pero al mismo 
tiempo percibe la tarea como razonablemente desafiante. Por lo que, la tarea no debe ser muy fácil, ni muy compleja. Esto hace reflexionar sobre parcializar y basar el proceso en un aprendizaje colaborativo, con la adecuada vigilancia, guía y retroalimentación, manteniendo las emociones positivas como factor intrínseco fundamental en el desarrollo de la tarea.

\section{Referencias}

Cabanach, R., Souto-Gestal, A., \& Franco, V. (2016). Escala de Estresores Académicos para la evaluación de los estresores académicos en estudiantes universitarios. Revista Iberoamericana de Psicología y Salud, 7(2), 41-50, ISSN: 2171-2069; e-ISSN: 1989-9246. Recuperado de: https://doi.org/10.1016/j.rips.2016.05.001

Celis, J., Bustamante, M., Cabrera, D., Cabrera, M., Alarcón, W., \& Monge, E. (2001). Ansiedad y Estrés Académico en Estudiantes de Medicina Humana del Primer y Sexto Año. Anales de la Facultad de Medicina, 62(1), 25-30, ISSN: 1025-5583; e-ISSN: 1609-9419. Recuperado de: https://doi.org/10.15381/anales.v62i1.4143

Diamond, A. (2013). Executive Functions. Annual Review of Psychology, 64, 135-168, e-ISSN: 0066-4308. Recovered from:

https://doi.org/10.1146/annurev-psych-113011-143750

Elizondo, A., Rodríguez, J., \& Rodríguez, I. (2018). La importancia de la emoción en el aprendizaje: Propuestas para mejorar la motivación de los estudiantes. Cuaderno de Pedagogía Universitaria, 15(29), 311, ISSN: 1814-4144; e-ISSN: 1814-4152. Recuperado de:

https://doi.org/10.29197/cpu.v15i29.296

Halkjelsvik, T., \& Rise, J. (2014). Persistence Motives in Irrational Decisions to Complete a Boring Task. Personality and Social Psychology Bulletin, 41(1), 90-102, e-ISSN: 1552-7433. Recovered from:

https://doi.org/10.1177/0146167214557008 
Hiramatsu, Y., Sato, F., Ito, A., Hatano, H., Sato, M., Watanabe, Y., \& Sasaki, A. (2017). Designing Mobile Application to Motivate Young People to Visit Cultural Heritage Sites. International Journal of Business, Human and Social Sciences, 11(1), 121-128, e-ISSN: 2517-9411. Recovered from: https://doi.org/10.5281/zenodo.1339650

Kupor, D., \& Tormala, Z. (2015). Persuasion, Interrupted: The Effect of Momentary Interruptions on Message Processing and Persuasion. Journal of Consumer Research, 42(2), 300-315, e-ISSN: 1537-5277. Recovered from: https://doi.org/10.1093/jcr/ucv018

Li, H., Li, F., \& Chen, T. (2018). A motivational-cognitive model of creativity and the role of autonomy. Journal of Business Research, 92, 179-188, e-ISSN 0148-2963. Recovered from:

https://doi.org/10.1016/j.jbusres.2018.07.025

Lang, P., \& Davis, M. (2006). Emotion, motivation, and the brain: Reflex foundations in animal and human research. Progress in Brain Research, 156, 3-29, e-ISSN: 0079-6123. Recovered from: https://doi.org/10.1016/S0079-6123(06)56001-7

Manes, F., \& Niro, M. (2014). Usar el cerebro. Conocer nuestra mente para vivir mejor. Buenos Aires, Argentina: Editorial Planeta.

Morgane, P., Galler, J., \& Mokler, D. (2005). A review of systems and networks of the limbic forebrain/limbic midbrain. Progress in Neurobiology, 75(2), 143-160, e-ISSN: 1873-5118. Recovered from: https://doi.org/10.1016/j.pneurobio.2005.01.001

Ovsiankina, M. (1928). Untersuchungen zur Handlungs- und Affektpsychologie. Psychologische Forschung, 11, 302-379, ISSN: 0033-3026. Germany: Bernhard Hommel Institute of Psychology Leiden University.

Pesántez, L. (2020). Los Grupos de Innovación Educativa GIE: Una alternativa válida para los Docentes Universitarios. Revista 
Scientific, 5(15), 10-22, e-ISSN: 2542-2987. Recuperado de:

https://doi.org/10.29394/Scientific.issn.2542-2987.2020.5.15.0.10-22

Portilla, G., \& Leyva, A. (2018). Innovación de la Práctica Docente en la UNAE, Ecuador recurriendo a la Web 2.0. Revista Scientific, 3(7), 140-154, e-ISSN: 2542-2987. Recuperado de:

https://doi.org/10.29394/Scientific.issn.2542-2987.2018.3.7.7.140-154

Schaufeli, W., Martínez, I., Marques, A., Salanova, M., \& Bakker, A. (2002). Burnout and Engagement in University Students: A Cross-National Study. Journal of Cross-Cultural Psychology, 33(5), 464-481, e-ISSN: 1552-5422. Recovered from:

https://doi.org/10.1177/0022022102033005003

Shin, S., \& Zhou, J. (2017). Transformational Leadership, Conservation, and Creativity: Evidence from Korea. Academy of Management Journal, 46(6), 703-714, e-ISSN: 1948-0989. Recovered from:

https://doi.org/10.5465/30040662

Verdejo-García, A., \& Bechara, A. (2010). Neuropsicología de las Funciones

Ejecutivas. Psicothema, 22(2), 227-235, e-ISSN: 0214-9915.

Recuperado de: https://www.redalyc.org/articulo.oa?id=72712496009

Wilkie, K. (2016). Rise or Resist: Exploring Senior Secondary Students' Reactions to Challenging Mathematics Tasks Incorporating Multiple Strategies. Eurasia Journal of Mathematics, Science and Technology Education, 12(8), 2061-2083, e-ISSN: 1305-8223.

Recovered from: https://doi.org/10.12973/eurasia.2016.1260a

Zeigarnik, B. (1927). Das Behalten erledigter und unerledigter Handlungen.

Psychological Research, 1-85, ISSN: 0340-0727. Germany: Aus dem Psychologischen Institut der Universität Berlin. 


\section{Ximena Paz Martínez Oportus \\ e-mail: ximena.martinez@umayor.cl}

Nacida en Concepción, Chile, el 6 de junio del año 1977.

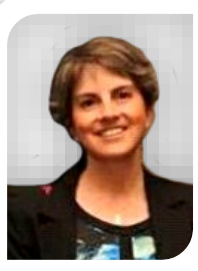

Médico Veterinario; Magister en Ciencias Veterinarias de la Universidad de Chile (UCHILE); Magister en Dirección de Instituciones de Salud, por la Universidad Mayor (UM);

Dieciocho años de experiencia clínica y de Docencia Universitaria; Diplomada en Docencia Efectiva, en Psicología Budista y en Metodología de Investigación Cuantitativa; Experiencia docente y de investigación en la Universidad de Chile (UCHILE), en la Facultad de Medicina Norte y la Facultad de Bioquímica de la Pontificia Universidad Católica de Chile (UC); Actualmente, docente de pregrado y postgrado en Neurociencias básicas y Neurociencias de la Educación; Directora Regional de Postgrados, en la Facultad de Ciencias, de la Universidad Mayor (UM), Temuco, Chile.

El contenido de este manuscrito se difunde bajo una Licencia de Creative Commons ReconocimientoNoComercial-Compartirlgual 4.0 Internacional 